\title{
Analysis of COVID-19 M Protein for Possible Clues Regarding Virion Stability, Longevity and Spreading
}

\author{
Saam Hasan ${ }^{1,2}$, Muhammad Maqsud Hossain ${ }^{1,2 \dagger}$ \\ ${ }^{1}$ Department of Biochemistry and Microbiology, \\ North South University, Dhaka, Bangladesh \\ ${ }^{2}$ NSU Genome Research Institute-NGRI, \\ North South University, Dhaka Bangladesh
}

\begin{abstract}
The Severe Acute Respiratory Syndrome Coronavirus 2 or COVID-19 has been the cause of a global pandemic in 2020. With the numbers infected rising well above a 100,000 and confirmed deaths above 4000, it has become the paramount health concern for the global community at present. The COVID-19 genome has since been sequenced and its predicted proteome identified. In this study, we looked at the expected COVID-19 proteins and compare them to its close relative, the Severe Acute Respiratory Syndrome-Related Coronavirus. In particular we focussed on the M protein which is known to play a significant role in the virion structure of Coronaviruses. The rationale here was that since the major risk factor associated with COVID-19 was its ease of spread, we wished to focus on the viral structure and architecture to look for clues that may indicate structural stability, thus prolonging the time span for which it can survive free of a host. As a result of the study, we found some rather interesting differences between the M protein for COVID-19 and the SARS-CoV virus M protein. This included amino acid changes from non-polar to polar residues in regions important for anchoring the protein in the envelope membrane.
\end{abstract}

Keywords: COVID-19, Proteomics, Systems Biology

\footnotetext{
*corresponding author

† E-mail: muhammad.maqsud@ @orthsouth.edu
} 


\section{Background}

The COVID-19 Novel Corona virus or Severe Acute Respiratory Syndrome Coronavirus 2 has been the cause of a worldwide pandemic in 2020. The novel strain, originating from Wuhan, China has as of March $12^{\text {th }}$, infected over 125,000 people and led to the deaths of over 4500 individuals worldwide. It has led to considerable disruptions in the economic and psychological welfare of populations, on top of the continuously increasing loss of life (1). Efforts at identifying possible solutions to combating the virus have thus far ranged from basic precautionary awareness campaigns to the suggestions of nucleotide based drugs currently being tested for other diseases as possible therapeutic agents (12). The genome of the COVID-19 novel coronavirus has been decoded and its expected proteome already established (RefSeq ID: NC_045512.2). However up until this point, to the best of our knowledge not much progress has been made in terms of identifying unique molecular features that are exclusive to this strain of the Coronavirus. Hence, in this study we made an attempt at studying the protein sequences of a relevant COVID-19 architectural protein in order to identify unique advantageous features. We chose to focus our efforts on the $\mathrm{M}$ Protein as it is the most abundant protein in the viral nucleocapsid and is believed to be responsible for maintaining the virion in its characteristic shape (7). This coincides with our investigative rationale; is there any particular feature that aids the spread of this strain. The overall mortality rate for the virus infection has been estimated to be around 3.6 percent as of this writing (14). Furthermore the primary susceptible groups are the immunocompromised. This leads us to believe that the primary focus needs to be on the mechanism of spreading, as the rapid rates at which this strain has infected communities across the world has arguably been its most dangerous aspect.

\section{Comparing the COVID-19 and SARS-CoV M Proteins}

The coronaviruses are known to contain four main structural proteins, the S, M, E, and N proteins (2). All of whom have been identified in the predicted proteome of COVID-19. The $S$ protein is the spike protein, responsible for gaining entry into the ER following infection (9, $10)$. The $\mathrm{N}$ protein resides inside the nucleocapsid and is an RNA binding protein $(6,11)$. The $\mathrm{M}$ and $\mathrm{E}$ proteins are both transmembrane proteins. The $\mathrm{M}$ protein is particularly significant as it is believed to be responsible for maintaining and giving the virion its shape (4).

It is a three pass membrane protein with three transmembrane domains. We obtained the protein sequences for the SARS Related Coronavirus as well as COVID-19 and did a multiple sequence alignment using NCBI Blast (8). Figure 1 shows the results of the alignment. Afterwards we took the sequences from the both proteins and attempted to predict their structures using SWISS-MODEL (3). Lastly we looked up known protein domains and epitopes within the SARS Related Coronavirus M Protein and scrutinized for any changes in those regions for the COVID-19 M Protein. The most significant epitope we found and considered was the Mn2 epitope that binds to human Cytotoxic T Lymphocytes (5). 


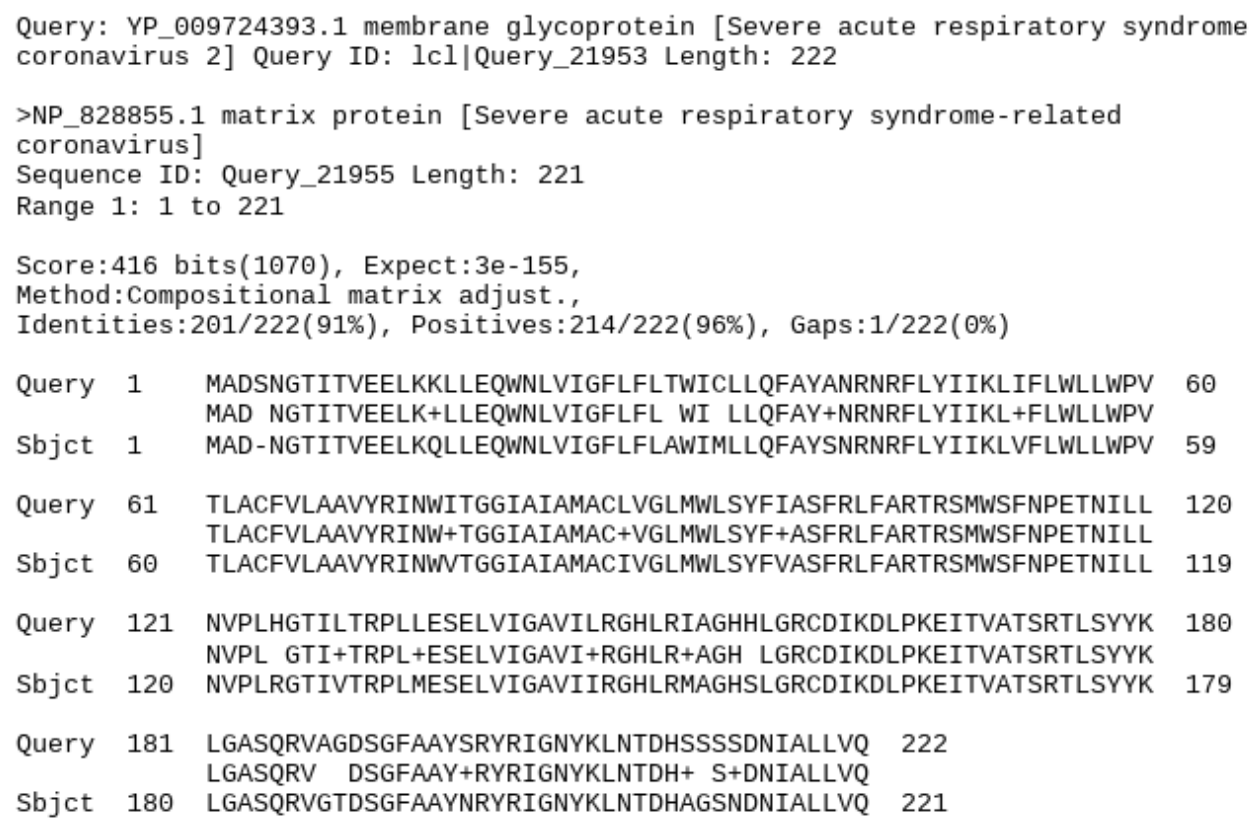

Figure 1: BLAST Multiple Sequence Protein alignment of COVID-19 M Protein with SARSCoV M Protein.

As figure 1 shows, there were a total of 20 mismatches and 1 gap between the two sequences. What caught our eye most was the fact that there were multiple amino acid substitutions in the transmembrane domains where bulkier non-polar amino acids in the SARS-CoV gene were replaced by more polar or less bulky ones in the COVID-19 protein. In addition, we found a serine inserted into position 4 of the COVID-19 M protein which its SARS Related Coronavirus counterpart did not have.

\section{Analysing the Structural and Functional Implications}

Serine provides an extra $\mathrm{OH}$ group near the $\mathrm{N}$ terminal end of the protein which we know makes up its ectodomain that is glycosylated (7). Coming over to the transmembrane regions, at position 33 the COVID-19 has a Cysteine, the equivalent position on SARS-CoV is occupied by a Methionine. At position 30, COVID-19 contains a Threonine, the corresponding position on SARS-CoV has an Alanine. Then at position 76 on the second transmembrane domain, the SARS-CoV protein contained a valine, compared to an Isoleucine on the COVID-19 protein. Starting from the latter, Isoleucine has a bulkier sidechain compared to valine, which in the hydrophobic interior of an envelope membrane could produce more hydrophobic interactions with the fatty acid chains of the lipid bilayer, possibly stabilizing the membrane to a greater degree. The previous couple of changes, the Met to Cys and Ala to The substitutions would seem more curious. Both would replace nonpolar sidechains with polar groups. This may seem counterintuitive for stabilizing a membrane structure, however crucially perhaps, this could theoretically allow for inter-chain 
bonds between protein molecules. The $\mathrm{OH}$ group introduced by Threonine could contribute to hydrogen bonding, while the thiol group introduced by Cysteine could give rise to a disulphide bond. We believe this may hold special importance, especially since our modelling run predicted the COVID-19 M Protein to form homo-trimers, whereas the SARS-CoV M protein is known to form homo-dimers (15). The latter was backed up when ran the SWISSMODEL tool on the amino acid sequence from the SARS-CoV protein for which the first predicted structure was a homo-dimer. Figure 2A and 2B show the predicted structures for COVID-19 and SARS-CoV M Protein respectively.

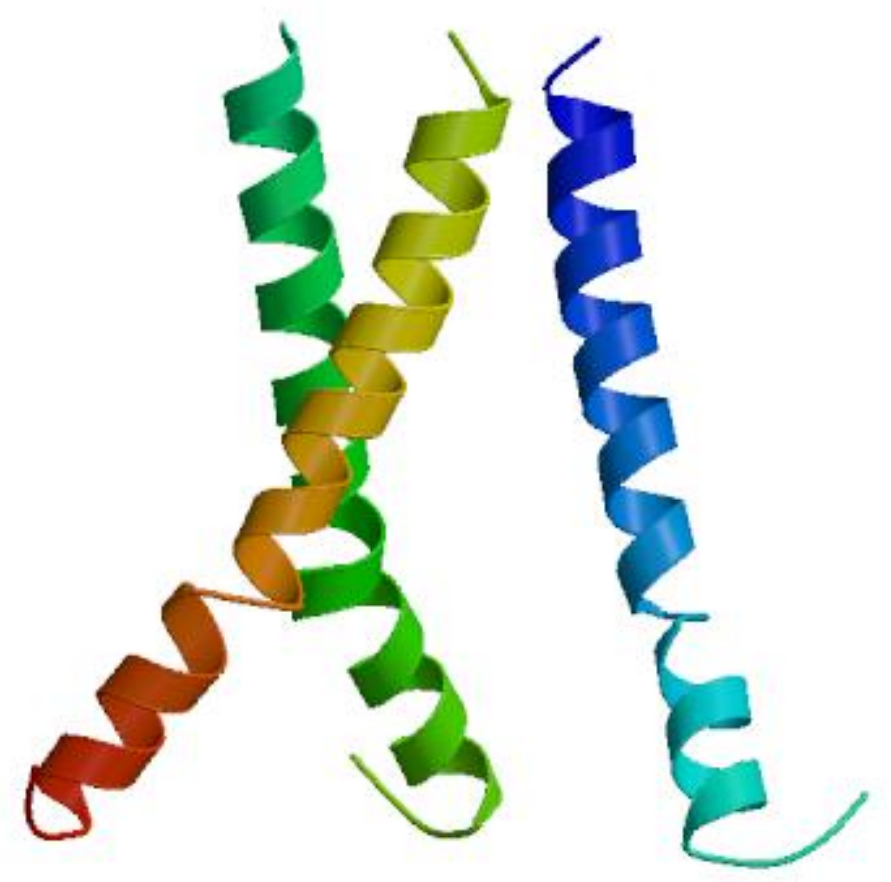

Figure 2A: Predicted homo-trimer protein structure for COVID-19 M Protein. 


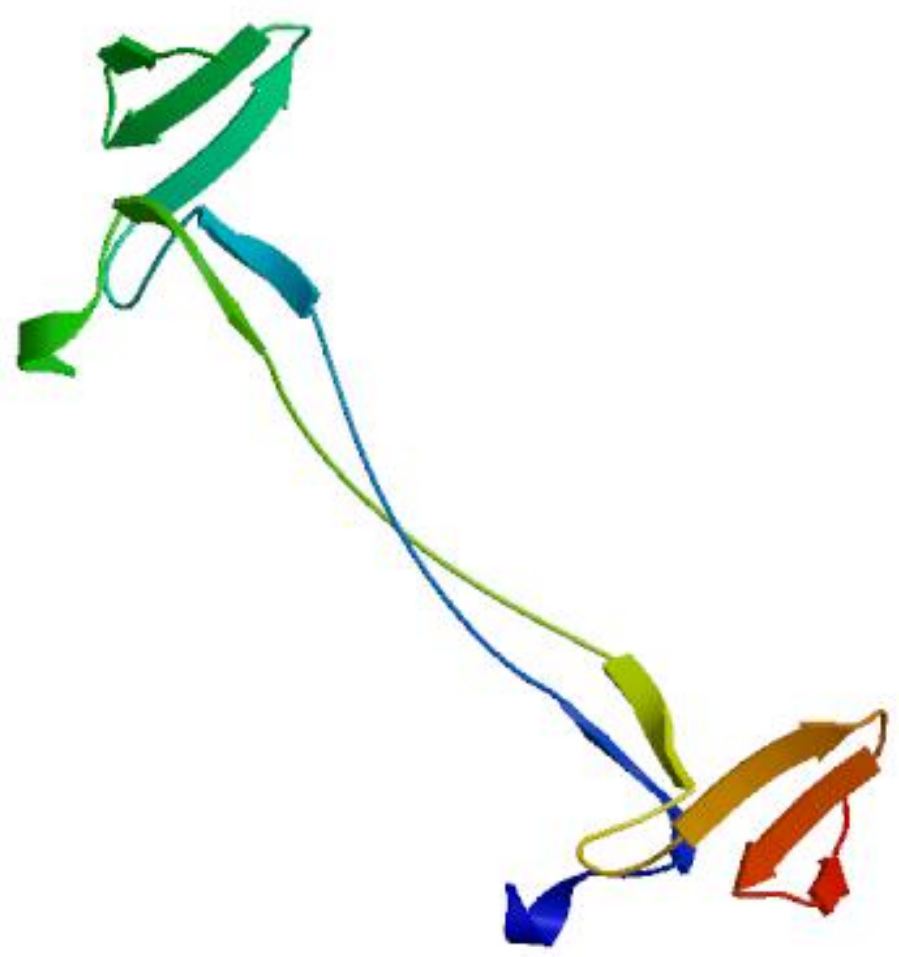

Figure 2B: Predicted homo-dimer protein structure for SARS-CoV M Protein.

\section{Discussion}

This leads us to a possible answer to the question we posed at the very start. We believe there might be a possibility of additional bonding interactions in the COVID-19 M protein that allows its structure to remain more stable and survive for longer. In general, it is what one would expect to happen with the introduction of additional protein-protein bonding, as per the usual biochemical make-up of lipid bilayer membranes and transmembrane proteins that pass through them (16). The Serine at the $\mathrm{N}$ terminus is another interesting observation. The $\mathrm{N}$ terminal of this protein is expected to be on the outside of the envelope (7) and possibly be exposed to the air and outside interactions.

Something that has come up in recent news reports is the ability of the COVID-19 viral particles to survive for longer on metal surfaces, although the actual study has not yet been published (18). Metals have lattice structures where the positive ions are in the middle and the electrons delocalised and free to move about (17). A polar group such as an $-\mathrm{OH}$ where the hydrogen atom would likely have a partial positive charge, may open the possibility for some kind of bio-electrostatic association that could help the virion to adhere to these surfaces. Although we are not sure as to how or if it could preserve the architectural integrity of the virion.

The major difference between the SARS-CoV and the COVID-19 outbreaks is the spread of the latter. SARS-Cov only effected 26 counties, with around 8000 confirmed cases (13). 
Granted, this needs to factor in the increased air travel in recent times, which, as per Statista is almost double it was in 2003 when the SARS epidemic happened. The information for that can be found here; https://www.statista.com/statistics/564769/airline-industry-number-offlights. Nonetheless, based on evidence and our molecular level analysis, we believe the $\mathrm{M}$ protein should be a candidate into future investigations that could shed more light on how the COVID-19 virus survives and functions. 


\section{References}

1. Chen Q, Quan B, Li X, Gao G, Zheng W, Zhang J et al. A report of clinical diagnosis and treatment of 9 cases of coronavirus disease 2019. Journal of Medical Virology. 2020;.

2. Fehr A, Perlman S. Coronaviruses: An Overview of Their Replication and Pathogenesis. Coronaviruses. 2015;:1-23.

3. Biasini M, Bienert S, Waterhouse A, Arnold K, Studer G, Schmidt T et al. SWISSMODEL: modelling protein tertiary and quaternary structure using evolutionary information. Nucleic Acids Research. 2014;42(W1):W252-W258.

4. Armstrong J, Niemann H, Smeekens S, Rottier P, Warren G. Sequence and topology of a model intracellular membrane protein, E1 glycoprotein, from a coronavirus. Nature. 1984;308(5961):751-752.

5. Liu J, Sun Y, Qi J, Chu F, Wu H, Gao F et al. The Membrane Protein of Severe Acute Respiratory Syndrome Coronavirus Acts as a Dominant Immunogen Revealed by a Clustering Region of Novel Functionally and Structurally Defined Cytotoxic T-Lymphocyte Epitopes. The Journal of Infectious Diseases. 2010;202(8):1171-1180.

6. Chang C, Sue S, Yu T, Hsieh C, Tsai C, Chiang Y et al. Modular organization of SARS coronavirus nucleocapsid protein. Journal of Biomedical Science. 2005;13(1):59-72.

7. Nal B, Chan C, Kien F, Siu L, Tse J, Chu K et al. Differential maturation and subcellular localization of severe acute respiratory syndrome coronavirus surface proteins S, M and E. Journal of General Virology. 2005;86(5):1423-1434.

8. Altschul S, Gish W, Miller W, Myers E, Lipman D. Basic local alignment search tool. Journal of Molecular Biology. 1990;215(3):403-410.

9. Beniac D, Andonov A, Grudeski E, Booth T. Architecture of the SARS coronavirus prefusion spike. Nature Structural \& Molecular Biology. 2006;13(8):751-752.

10. Delmas B, Laude H. Assembly of coronavirus spike protein into trimers and its role in epitope expression. Journal of Virology. 1990;64(11):5367-5375.

11. Hurst K, Koetzner C, Masters P. Identification of In Vivo-Interacting Domains of the Murine Coronavirus Nucleocapsid Protein. Journal of Virology. 2009;83(14):7221-7234.

12. Martinez M. Compounds with therapeutic potential against novel respiratory 2019 coronavirus. Antimicrobial Agents and Chemotherapy. 2020;.

13. WHO | SARS (Severe Acute Respiratory Syndrome) [Internet]. Who.int. 2020 [cited 13 March 2020]. Available from: https://www.who.int/ith/diseases/sars/en/

14. [Internet]. Who.int. 2020 [cited 13 March 2020]. Available from: https://www.who.int/docs/default-source/coronaviruse/20200312-sitrep-52-covid19.pdf?sfvrsn=e2bfc9c0_2

15. Godet M, L'Haridon R, Vautherot J, Laude H. TGEV corona virus ORF4 encodes a membrane protein that is incorporated into virions. Virology. 1992;188(2):666-675. 
16. Alberts B. Molecular biology of the cell. New York: Garland Science Taylor \& Francis; 2008 .

17. Tro N. Principles of chemistry.

18. News A. COVID-19 can last a few days on surfaces, according to new experiment findings [Internet]. ABC News. 2020 [cited 14 March 2020]. Available from: https://abcnews.go.com/Health/covid19-days-surfaces-experimentfindings/story?id=69569397 